\title{
MATERIALES ROMANOS, PROCEDENTES DE "LUCUS ASTURUM», DE RECIENTE INGRESO EN EL MUSEO DE OVIEDO
}

\author{
MATILDE ESCORTELL
}

\section{Antecedentes}

Lucus Asturum fue, sin duda, la ciudad más importante, en la antigüedad, dentro del territorio que hoy ocupa Asturias. Todos los indicios, hasta el momento, localizan a "Lucus Asturum" en la parroquia de Lugo en el concejo de Llanera; Lugo es hoy un importante centro ferroviario en la línea Madrid-Gijón.

La primera cita histórica se debe a Ptolomeo, que al consignar en sus Tablas las ciudades y pueblos de los astures, la cita con el nombre de Lucus Asturum y la denomina polis. En el siglo VII el Anónimo de Ravena habla de Luco Astorum entre una serie de civitates en torno a Bracara Augusta, tomadas de los itinerarios romanos; las civitates del Anónimo de Ravena son lo que los romanos llamaban mansiones; por lo tanto Lucus Asturum ya era centro importante de comunicaciones en época romana.

José Manuel González en su trabajo "Lucus Asturum» (1) argumenta toponímica y documentalmente, con toda precisión, la localización de este núcleo de población en Lugo de Llanera.

Las referencias sobre hallazgos arqueológicos en Lugo de Llanera son abundantes. Carvallo (2) dice de Lucus "que se hallan restos y edificios por aquellos campos». Risco (3) habla de "rastros de la ciudad». Cean Bermúdez (4) alude a las murallas y dice: "de ellas y de la población romana no ha quedado más que los cimientos, trozos de columnas, acueductos de ladrillo y varias monedas de cobre, plata y estaño, con otras preciosidades». En la obra de Bellmunt y Canella (5) se habla del hallazgo de algunas monedas de plata y cobre, trozos de columna, pedazos de bóvedas, ladrillos circulares, fragmentos de mosaicos.

"Ciriaco Miguel Vigil recoge el informe hecho en 1848 por el alcalde de Llanera, Ramón Ablanedo, a la Comisión Provincial de Monumentos, sobre los «vesti-

(1) González, M. J.: "Lucus Asturum», Valdediós, 1960, pág. 33, Oviedo, 1960.

(2) Carvallo, L. A. de: "Antigüedades y cosas memorables del Principado de Asturias», Madrid, 1965.

(3) Risco: «España Sagrada», t. XXXVII, Madrid, 1787.

(4) Ceán Bermúdez: "Sumario de Antigüedades romanas que hay en España», Burgos, 203, Madrid, 1832.

(5) Bellmunt y Canella: "Asturias», t. III, pág. 243, Gijón, 1900. 
gios que a cada paso se encuentran en las inmediaciones de la iglesia parroquial de Lugo, es donde los labradores hallan con frecuencia ladrillos perfectamente calcinados y piedras labradas con arte, una de ellas con varias inscripciones, cuyo paradero se ignora; lo propio que el de las figuritas de bronce, descubiertas modernamente, representando madonas desnudas de cintura arriba, dos medallas con inscripciones desconocidas y esqueletos perfectamente exhumados». El autor de este informe hizo donación al Museo Provincial de "varias piedrecitas de colores en forma de cubos que fueron levantadas con el arado en término de aquella parroquia" (6).

Elías Quiros Tuñón hace referencia al fuste de columna roto en dos partes, que tenía inscripción, hallado en Lugo de Llanera: «En Lugo había el año 52 un fuste de columna, que me pareció hablaba de Numeriano, pero no tuve tiempo para copiar la inscripción" (7). Constantino Cabal (8) recoge la inscripción de ambos fragmentos: M[A]RCO [A(urelio) N]UMERIO / [NUM]ERIANO NOBI / [LIS]SIMMO CAESARI. En el otro fragmento ..IA.. / ..RIO.. / ..NLIO.. / ..ON. Cabal relaciona esta inscripción con un miliario de Numeriano, hijo del emperador Caro, que tomó el título de Caesar en el año 282; miliario de la vía de Asturica a Lucus Augusti, pasando por Lucus Asturum.

Se tiene noticias de que en los años 40 se realizaron excavaciones junto a la desaparecida iglesia de Santa María de Lugo de Llanera, pero los resultados son bastante confusos. En las excavaciones que practicaron D. José Cuesta Fernández y D. José Fernández Menéndez fueron hallados restos de un pavimento de ladrillos romboidales, así como ladrillos circulares, propios de las suspensurae de Hipocausto, que se encuentran en el Museo Arqueológico (9).

La Universidad de Oviedo realizó excavaciones en Lugo de Llanera en 1981. Armando Fernández (10) ha estudiado y publicado la terra sigillata hallada en esta excavación. Dice textualmente: "la excavación se realizó en la finca donde estuvo enclavada la antigua iglesia de Santa María de Lugo, junto al cementerio viejo por su sector Este, mientras por el Norte un camino vecinal lo separa del que llaman en el lugar " "prado de Lugo" muy próximo al actual Lugo de Llanera". La falta de una memoria de la citada excavación priva al lector de un marco arqueológicoexplicativo y en espera de su publicación, trataré de esbozar brevemente, amén del problema dialéctico que plantea la falta de ilustración gráfica del mismo. Se abrieron tres catas de $3 \times 3$ y dos de $3 \times 6$, en las que tras un nivel de superficie con una potencia de $30 \mathrm{~cm}$., aproximadamente, al que el haber sido tierra de labrantío le da cierta homogeneidad, se suceden los niveles rotos y alterados, cuya explicación hay que buscar en la función que el lugar desempeñó, que no fue otra

(6) Diego Santos, F.: “Historia de Asturias”, t. 3, pág. 121, Ediciones Ayalga, 1973.

(7) Tuñón Quirós: “Memoria sobre la guerra que los romanos hicieron en Asturias”, pág. 10, Oviedo, 1856 .

(8) Cabal, C.: “Alfonso II el Caston, pág. 182, Oviedo, 1943.

(9) Escortell, M.: "Catálogo de las salas de cultura romana del Museo Arqueológico de Oviedo", Oviedo, 1975

(10) Fernández, A.: "Cerámica romana, terra sigillata en Lugo de Llanera». Boletín Inst. Estudios Asturianos, n. ${ }^{\circ} 108$, pág. 45, Oviedo, 1983. 
que la de cementerio, con lo cual la estratigrafía queda interrumpida frecuentemente, con lo cual toda valoración cronológica basada en el método estratigráfico queda descartada".

Las cerámicas estudiadas por Armando Fernández son fragmentos de terra sigillata, lisa y decorada; de formas lisas la Drag. 15/17, Drag. 24/25 y Drag. 36; en formas decoradas Drag. 29, Drag. 30 y Drag. 29/37. Estas cerámicas, aunque nos pueden dar una fecha inicial, para el yacimiento en la segunda mitad del siglo I, son productos que perduran hasta el siglo IV.

En el mes de agosto de 1984, con motivo de unas obras, que RENFE realizó en la estación de Lugo de Llanera aparecieron restos arqueológicos, que fueron detectados por José Luis Maxide Carariego, quien hizo entrega de ellos al Museo, materiales que constituyen un dato arqueológico más, para la localización de Lucus Asturum en Lugo de Llanera. El lugar exacto es frente al edificio y andén de la estación, al otro lado de la vía. Los materiales, que se recogieron son ladrillos circulares, fragmentos de ladrillos rectangulares y varios fragmentos de mosaico.

\section{Ladrillos}

Ladrillo circular de suspensurae de hipocausto de unas termas. Dimensiones: diámetro: $18 \mathrm{~cm}$.; altura: $8 \mathrm{~cm}$. (Foto 1).

Ladrillo circular de suspensurae de hipocausto de unas termas; tiene el borde estriado y conserva restos de la argamasa de sujeción a otro ladrillo. Dimensiones: diámetro: $22 \mathrm{~cm}$; altura: $7 \mathrm{~cm}$. (Foto 1).

Ladrillo circular de suspensurae de hipocausto de unas termas. Dimensiones: diámetro: $23 \mathrm{~cm}$; altura: $8 \mathrm{~cm}$.

Fragmento de ladrillo rectangular. Dimensiones: ancho: $29,5 \mathrm{~cm}$.; largo: $26 \mathrm{~cm}$. lo conservado, que es aproximadamente la mitad (Foto 1).

\section{Fragmentos de mosaico (Foto 2)}

Aunque no hemos podido conseguir recomponer una superficie mínima del mosaico, por los fragmentos que aquí reseñamos podemos asegurar se trataba de un mosaico de decoración geométrica, de época tardía, como otros ya conocidos en Asturias.

Lo que estos fragmentos conservan del lecho es una capa de cal con polvo de ladrillo y otra capa de cal sobre las que se asientan las teselas. Las teselas tienen entre 6 y $8 \mathrm{~mm}$. de arista y los colores son: negro, blanco, rojo, gris y ocre.

Presentamos gráficamente aquellos fragmentos, en los que se puede intuir el motivo decorativo. En uno de ellos se aprecia perfectamente un ajedrezado, alternando un cuadro de teselas rojas y otro en que aparecen mezcladas teselas grises y ocres; este ajedrezado está bordeado por una línea de teselas negras y dos líneas de teselas blancas. En otros tres fragmentos la típica trenza romana de época tardía 


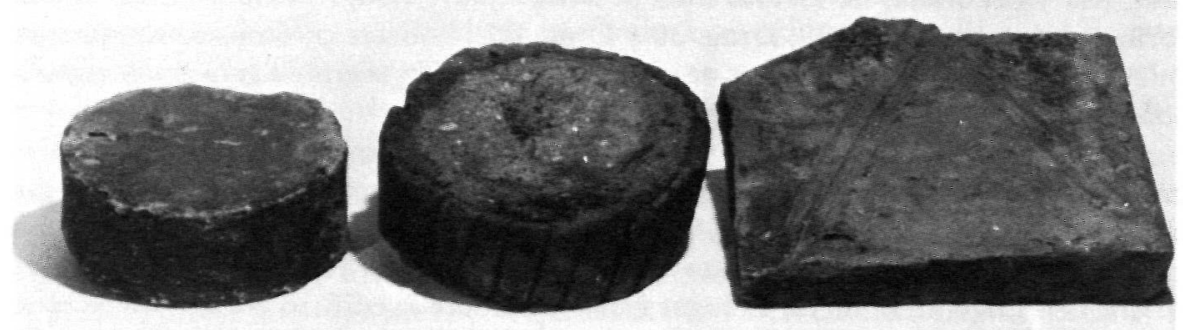

FOTO. 1.-Ladrillos procedentes de Lucus Asturum.

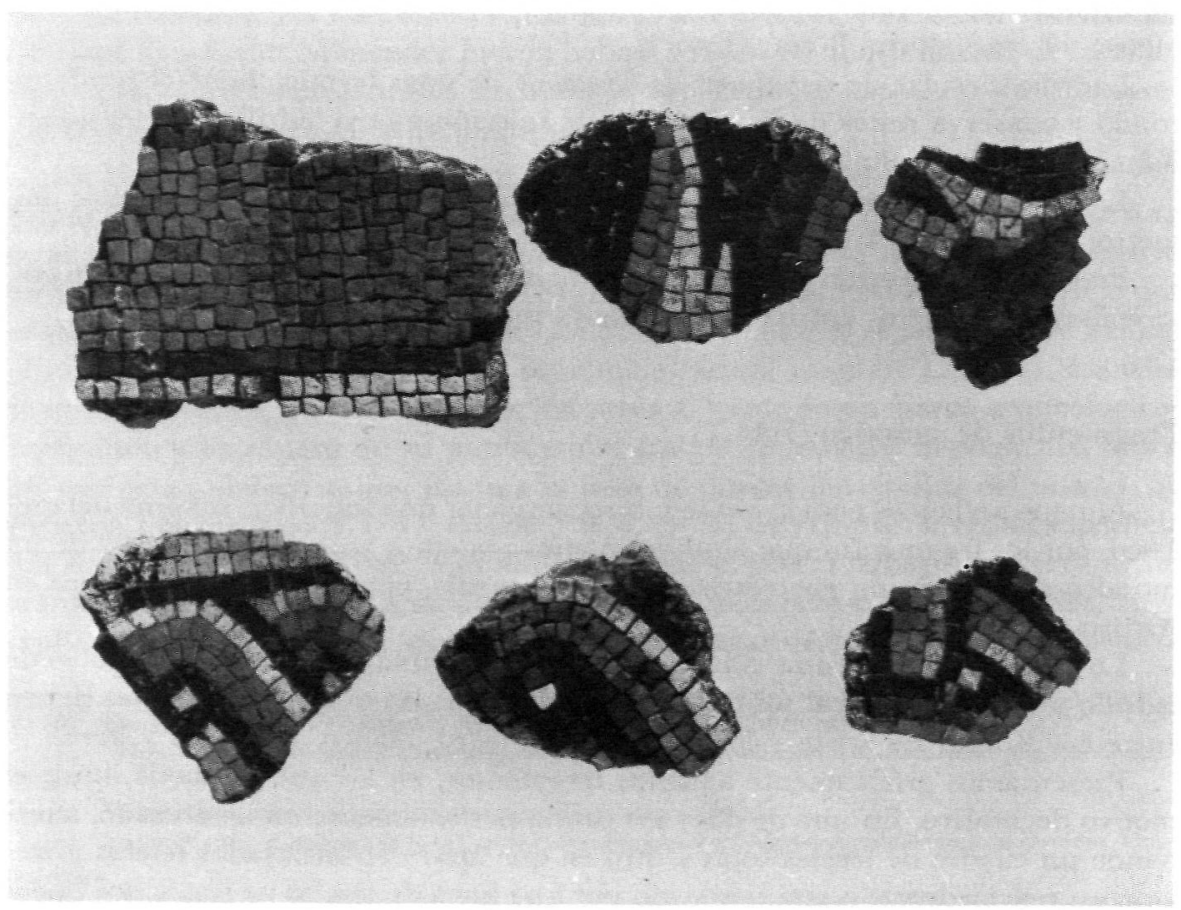

Foto 2.-Fragmentos de mosaico procedentes de Lucus Asturum. 
es fácilmente reconocible, aunque la combinación de colores es distinta en los tres. Y por último hemos escogido dos fragmentos, cuya decoración se podría interpretar como un motivo vegetal estilizado.

Con esta muestra creo no es difícil imaginar un mosaico con una orla de ajedrezado y el campo dividido, por medio de la típica trenza romana en varios compartimentos, ocupados por motivos diversos.

Para una cronología de estos restos de mosaico de Lugo de Llanera, por el momento, hemos de acudir a otros mosaicos ya conocidos, como los de Vega del Ciego, Andallón y Veranes.

Mosaico de Vega del Ciego. Las ruinas de esta villa se encuentran exactamente en la Eria de Vidriales, al O. de Vega del Ciego (Lena). La finca donde apareció el mosaico se denomina "El Fabón" situada a unos $500 \mathrm{~m}$. al O. de la carretera general Oviedo-León y a unos $70 \mathrm{~m}$. al N. del arroyo de Sorribas, es decir en la antigua vía de Asturica a Lucus Augusti, pasando por Lucus Asturum. Un topónimo muy próximo a las ruinas, Memorana, nos da el posible nombre de su dueño: Memorius. El Cosmógrafo Anónimo de Ravena menciona en el siglo VII un topónimo Memoriana, como lugar situado entre Legio y Lucus Asturum. Es muy posible que las ruinas de Vega del Ciego correspondan a dicho lugar.

El mosaico fue estudiado y publicado por D. Manuel Jorge Aragoneses (11). Este mosaico de unos 6,5 m. de lado es el pavimento de una de las habitaciones, a la que se penetra desde un corredor longitudinal; en el lado Este del mosaico hay una zona saliente de la línea general de contorno, ocupada por una alfombrilla, decorada con motivos geométricos, que corresponde precisamente al umbral de la puerta, que da paso del corredor a la habitación.

El lecho en que se apoyaban las teselas estaba formado por las siguientes capas: un firme de hormigón de ladrillo y piedras de río, una capa de cal con abundante polvo de ladrillo; una capa de cal sobre la que se asientan las teselas. Estas tienen unos $8 \mathrm{~mm}$. de lado, siendo de varios colores: blancas, moradas, negras, amarillas, salmón y rojo. El contorno del mosaico es una zona de ajedrezado, interrumpida en los lados E.S. y O. por sendas alfombrillas con motivos geométricos; esta orla de ajedrezado está rodeada a su vez por una banda blanca, decorada en algún sector por crucetas negras.

Una triple línea blanca, negra y blanca separa la orla del campo. Los motivos, que se repiten alternativamente son siete: círculos concéntricos continuos en torno a un cuadrifolio; líneas estrelladas concéntricas con una cruz patada en el centro; un jarrón de panza gallonada; un pez en medio de moluscos; dos pájaros afrontados sobre sendas ramas; círculos concéntricos en sectores alternos en torno a un cuadrifolio; un motivo floral muy estilizado. Estos motivos se distribuyen con arreglo a una rigurosa composición geométrica; la superficie del campo está dividida por medio de la característica trenza romana en sesenta y cuatro espacios cuadrados, de los que se suprimen los cuatro centrales, para el emblema.

(11) Jorge Aragoneses, $\mathrm{M} .:$ «El mosaico romano de Vega del Ciego (Asturias)». Boletín Inst. Estudios Asturianos, n. ${ }^{\circ}$ XXI, pág. 3, Oviedo, 1954. 
El estudio de estos motivos y su distribución llevan a Jorge Aragoneses a situar, cronológicamente, el mosaico de Vega del Ciego a finales del siglo IV y primeros decenios del V.

Mosaico de Andallón. Fue hallado en la finca El Pedregal, en el término de San Martín de Andallón, en el concejo de Las Regueras. Este mosaico ha sido estudiado y publicado por Juana Bellón (12); los motivos decorativos son geométricos y los colores de sus teselas son: blanco, negro, siena, amarillo y gris. La decoración está distribuida de la siguiente manera: a) cenefa exterior ornada de peltas amarillas enmarcadas en una línea de teselas negras y recortadas sobre fondo blanco, b) calles intermedias, formadas por "estrellas de rombos", que enmarcan grandes cuadrados, c) los grandes cuadrados están enmarcados en líneas quebradas, con los siguientes motivos en su interior: combinaciones de cuadrados; nudo salomónico de doble lazo; nudo salomónico de triple lazo; rosa cuadrifolia. Juana Bellón sitúa cronológicamente el mosaico de Andallón en el último tercio del siglo IV o principios del V. Sin embargo Carmen Fernández Ochoa (13) lo lleva a mediados del siglo III o como mucho principios del IV.

Mosaico de Veranes. Fue dado a conocer por Valdés Gutiérrez (14) en 1954. Apareció en el lugar donde hoy se encuentran los restos de la llamada basílica de Sta. María de Veranes y fue trasladado a la abadía de Cenero (Gijón) en la que se hizo una reconstrucción ideal por Pedro Hurlé.

Manuel Valdés Gutiérrez dice que: «imitaba un tablero de ajedrez con cuadros de tamaño de un tablero ordinario, coloreados de rojo, blanco y azul, alternando unos con otros, colocando el mosaico por los mismos procedimientos de hoy, sobre una capa de argamasa hecha de polvo de ladrillo y lechada de cal, y sus piedrecitas perfectamente alineadas que forman una superficie completamente plana».

Según la reconstrucción, que hay en la abadía de Cenero se trata de un gran octógono, flanqueados en sus lados por trapecios, separados éstos entre sí y del octógono central por medio de la típica trenza romana.

Se trata de una pieza tardorromana; no conviene olvidar, que muy próximo al lugar donde fue hallado se encuentra una villa, en Murias de Beloño, cuya ocupación romana está atestiguada hasta el siglo IV.

Es inevitable asociar los restos del mosaico de Lugo de Llanera con los tres que hemos descrito, pero muy especialmente con el mosaico de Vega del Ciego, aunque la factura es mucho más cuidada, en todos los aspectos, en el mosaico de Lugo de Llanera.

(12) Bellon, J.: “Nuevas aportaciones a la arqueología romana de Asturias". Boletín Inst. Estudios As-

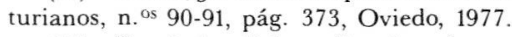

(13) Fernández Ochoa, C.: "Asturias en la época romana", pág. 360, Madrid, 1982.

(14) Valdés Gutiérrez, M.: “El mosaico romano de Santa María de Veranes”, Diario El Comercio, Gijón, 20 octubre, 1954. 